\title{
Enzymatic Inhibition of Phytochemical from Garcinia imberti on Homology Modelled Beta-lactamase Protein in Staphylococcus sciuri
}

\author{
Srikrishnan Rajendran Sriramkumar, ${ }^{1, *}$, Natesan Sivakumar ${ }^{2}$, Ponnuswamy Vijayaraghavan ${ }^{3}$ \\ 'Department of Microbiology, Bharathiyar University, Coimbatore, Tamil Nadu, INDIA. \\ 2Department of Molecular Microbiology, Madurai Kamaraj University, Madurai, Tamil Nadu, INDIA. \\ ${ }^{3}$ Bioprocess Engineering Division, Smykon Biotech Pvt. Ltd., Nagercoil, Kanyakumari District, Tamil Nadu, INDIA.
}

\begin{abstract}
Objectives: To analyze the interactions of modeled Beta-lactamase from Staphylococcus sciuri with phytochemical compounds from medicinal plant, Garcinia imberti in the docked complex. Methods: The protein-protein blast (BLASTP) analysis of target sequence of Beta-lactamase protein from Staphylococcus sciuri, against protein data bank (PDB) resulted that the X-ray crystal structure of beta-lactamase from Staphylococcus aureus was carried out. The sequence alignment was performed to build the initial model of Beta-lactamase protein from Staphylococcus sciuri using Modeler $9 \mathrm{v} 9$ by applying spatial restraints from the initial structure, a bundle of 3 models were developed using random generation for further analysis. The Ramachandran plot of the energy minimized model of Beta-lactamase protein from Staphylococcus sciuri was also carried out. The modeled Betalactamase protein from $S$. sciurii was subjected to difference of Gaussian (DoG) site scorer to predict the possible binding sites. Results: The results indicate that 4-Butylanisole and trans-9-Octadecene exhibited promising inhibitory activity. The docking studies also implies that the conserved amino acids Glutamine and Asparagine, Lysine and Phenyl alanine in the active site of beta-lactamase are critical in binding compounds with these
\end{abstract}

receptor. These docking interactions implies that the $=\mathrm{O}$ (keto group) present in the compounds and $\mathrm{NH}$ (amino group) on the amino acids favors the $\mathrm{H}$ bond interactions. Conclusion: The present findings throws light for the design of novel beta lactamase inhibitor compounds with antimicrobial activity envisages that the amino acids Glutamine $(\mathrm{Q})$ and Asparagine (N), Lysine (L) and Phenyl alanine (F) should be considered during its design for implying its action as a best antimicrobial compound to target $S$. sciurii.

Key words: Beta-lactamase, Docking, Phytochemicals, Staphylococcus sciuri, Garcinia imberti.

Correspondence

Dr. Srikrishnan Rajendran Sriramkumar

Department of Microbiology, Bharathiyar University, Coimbatore-641046, Tamil Nadu, INDIA.

Phone: +91 9442312911

Email: sriram.microbio@gmail.com

DOI: 10.5530/jyp.2020.12.8

\section{INTRODUCTION}

Root canal infection seems to be a major oral health problem throughout the world. It develops mainly due to the survival of microbes that are arising from endogenous source. The infected root canal system is capable of harbouring the pathogenic microbes. Also this system receives virulent product of microbes. These products are responsible for the development of apical periodontitis. ${ }^{1}$ Apical periodontitis have a polymicrobial etiology. $^{2}$ In this infection, Prevotella sp., Porphyromonas spp. Fusobacterium sp., Enterococus spp. and Candida spp are mostly involved. ${ }^{3}$ To eliminate these organism from the root canal, several irrigants like $\mathrm{NaOCl}$, Chlorohexidine and Hydrogen peroxide, ${ }^{4}$ nevertheless the efforts to be taken to treat this infection is failed frequently, due to procedural errors as well as capable of surviving multi drug resistant microbes within the root canal. Moreover the irrigant causes tissue damage and tissue necrosis. In order to prevent this, the present study helps to formulate a new suitable herbal irrigant for the successful endodontic treatment.

To fill the huge gap between the annotated sequence and 3D structures of the proteins, Theoretical or homology modeling serves as less expensive and faster computational resources to successfully determine the $3 \mathrm{D}$ structures of protein. ${ }^{5}$ Comparative modeling plays an important role in the absence of experimentally derives structures and remains as a viable cost-effective alternate for structure based drug designing. ${ }^{6}$ As the homology modeling builds the 3D structures of proteins based on template structures, it relies upon the percentage of sequence similarity and its accuracy in alignments. The accuracy of the built model always depends upon the choice of template, alignment accuracy and refinement of the model. Generally, the models built with the templates exhibiting over $70 \%$ identities are enough accurate for drug discovery applications. ${ }^{7}$

In line with this, various applications associated with the homology modeling, less expensive technique to generate reasonable accurate model, provides to identify the conserved regions that significantly helps to predict the functional sites and aid to trace the evolutionary relationship between sequences by multiple template alignments and also helps to identify the accurate binding modes to study the protein - ligand interactions and aid the mutagenesis studies. ${ }^{8}$ In general, the interaction between two molecules - the ligand and the protein that interacts with each other to form a complex is derived by means of certain forces at their point of intercept (binding sites of receptor). ${ }^{9-11}$ The fitness function describes the interactions between the ligand and the receptor. Docking conventionally reports two important information such as correct conformation of a ligand-receptor complex and its binding affinity which represents an approximation of the binding free energy relevant to the formation of complex. ${ }^{12}$

Thus in this present study the homology modeling of Beta-lactamase protein from Staphylococcus sciuri and docking studies of phytochemical compounds of methanol extract revealed through gas chromatography - 
mass spectrophotometry (GC-MS) analysis were carried out as it might lead to the design of novel antibiotic as a therapeutic agents in the form of irrigant against the troublesome multidrug resistant Staphylococcus sciuri for the successful endodontic treatment.

\section{MATERIALS AND METHODS}

\section{Target sequence and potential template search}

The Beta-lactamase protein sequence of Staphylococcus sciuri was retrieved from the UniprotKB database (uniprot ID: U6EG14) $.^{13} \mathrm{NCBI}-$ BlastP (basic local alignment search tool) was used to search the homologous sequences against PDB and obtained homologous sequence was considered as the potential template structure for homology modeling. ${ }^{14}$ The atomic co-ordinate file of the template structure was obtained from the PDB. ${ }^{15}$ The sequence alignment and alignment errors were refined by using ClustalW program as homology modelling relies on the sequence alignment. ${ }^{16}$

\section{Homology modeling}

The automated homology modeling software Modeler9v9 was used to built the model based on the final sequence alignment file of target and the template sequence. ${ }^{17}$ The atomic coordinate file of the template structure was used to build the 3D model by generating the satisfaction of spatial restraints. ${ }^{18}$ A bundle of 3 models were calculated from the starting structure by random generation. The best model was selected based on the least root - mean - square deviation (RMSD) value. This best model was energy minimized by applying 20 steps of each steepest descent and conjugates gradient using GROMOS of SwissPDBviewer and was used for further analysis. ${ }^{19}$

\section{Model assessment}

The quality of the generated model was assessed by checking the stereo chemical parameters using a program to check the stereochemical quality of protein structures (PROCHECK), ${ }^{20}$ Verfiy $3 D^{21}$ and Errat ${ }^{22}$ at Save server. ${ }^{23}$

\section{Prediction of binding site}

To determine the binding affinities between modeled Beta-lactamase protein sequence of Staphylococcus sciuri and the phytochemical compounds identified through GC-MS were predicted through difference of Gaussian site (DoG) Site Scorer. ${ }^{24}$

\section{Ligand generation and flexible docking}

The 3D structure of identified phytochemical compounds from GC-MS analysis of Methanol extracts of Garcinia imberti was retrieved as SDFile from PubChem Database. The obtained SDF structures was docked with the amino acids in the predicted binding site of modeled Beta-lactamase protein from Staphylococcus sciuri using Flex $\mathrm{X}^{25}$ with following parameters i) default general docking information ii) base placement using triangle matching, iii) scoring of full score contribution and threshold of 0,30 and No score contribution and threshold of 0,70. iv) Chemical parameters of clash handling values for protein ligand clashes with maximum allowed overlap volume of $2.9 \mathrm{~A}^{03}$ and intra-ligand clashes with clash factor of 0.6 and considering the hydrogen in internal clash tests. v) Default docking details values of 200 for both the maximum number of solutions per iteration and maximum number of solutions per fragmentation.

\section{Prediction of legend- receptor interactions}

The interactions of phytochemical compounds with modeled Beta-lactamase protein from Staphylococcus sciuri in the docked complex were analyzed by the pose-view of LeadIT. ${ }^{26}$

\section{RESULTS}

The BLASTP analysis of target sequence of Beta-lactamase protein from Staphylococcus sciuri, against PDB resulted that the X-ray crystal structure of beta-lactamase from Staphylococcus aureus as the homologous sequences with sequence similarity of $71.6 \%$ at an E-value of $1.10 \mathrm{e}-20$. The template- target sequence alignment is shown in Figure 1. As both the sequences are of beta-lactamase and from same genera the resultant homologous sequence was selected as template structure for homology modeling. The sequence alignment file was used as input to build the initial model of Beta-lactamase protein from Staphylococcus sciuri using Modeler 9v9 by applying spatial restraints from the initial structure, a bundle of 3 models were developed using random generation and the best model was selected for further analysis based on its structural compatibility (structure with lowest Discrete Optimized Protein Energy (DOPE) score). The modeled structure was shown in Figure 2.

The overall stereo chemical quality of the model was assessed by PROCHECK, Verfiy3D and Errat of Saves server. The Ramachandran plot of the energy minimized model of Beta-lactamase protein from Staphylococcus sciuri showed $92.1 \%$ of the residues in the most favorable region, $7.0 \%$ in the additionally allowed region, $0.9 \%$ in the generously allowed region and $0.0 \%$ in the disallowed region (Figure 3 ). The Ramachandran plot of the all generated models of beta-lactamase Protein were analyzed and considered the best model as it exhibited more number of residues in the most favourable regions and also the low number of residues in disallowed region. The total quality G-factor was -0.13 . Further the overall quality factor and compatibility of an atomic model (3D) with amino acid sequence (1D) for the modelled Beta-lactamase protein from Staphylococcus sciuri was observed as 83.328 and $97 \%$ from ERRAT and Verify3D respectively and were given in Table 1 . The results of ERRAT and Verify-3D also confirm the model was reliable and of good quality.

The modeled Beta-lactamase protein from S. sciurii was subjected to DoG site scorer to predict the possible binding sites. The server revealed seven binding sites with their predicted Volume $\left[\AA^{3}\right]$, Surface $\left[\AA^{2}\right]$, Lipo surface $\left[\AA^{2}\right]$, Depth $[\AA]$ and Drug Score (Table 2). Among these, based on the predicted dock score it is considered that the P0 site with the highest dock score as the most potential binding site for the further docking studies.

The GC-MS analysis of methanol extracts of Garcinia imberti revealed the presence of nearly 250 compounds, among these compounds, the $3 \mathrm{D}$ conformations for only 83 compounds were able to generate. Thus, these 83 compounds were used to determine their inhibition activity against $S$. sciurii by revealing its binding efficiency through docking studies. The determined binding site $\mathrm{P} 0$ was considered as potential binding site five important compounds with generated 3D conformations (Figure 4). The best docking interactions score of $-22.1230 \mathrm{~kJ} / \mathrm{mol}$ was observed for the 1-butyl-4-methoxybenzene with the modeled Beta-lactamase protein from Staphylococcus sciurii. This interaction is favored by the formation of Hbond with Gln41 and hydrophobic interactions with Asp39, Phe126, Asn40, Trp65, Phe111, Thr109 and Lys67. It is observed that the standard

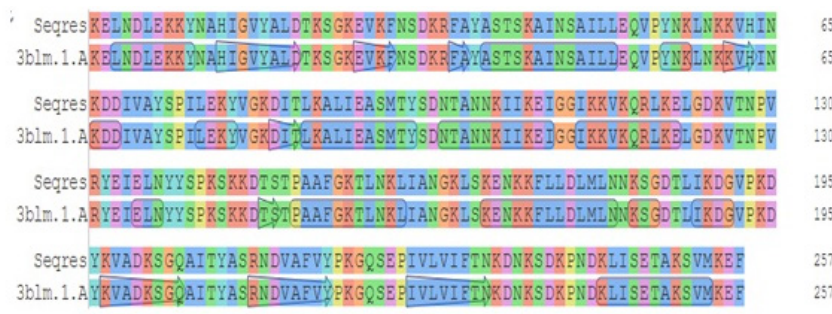

Figure 1: Template-target sequence alignment considered for homology modeling. 


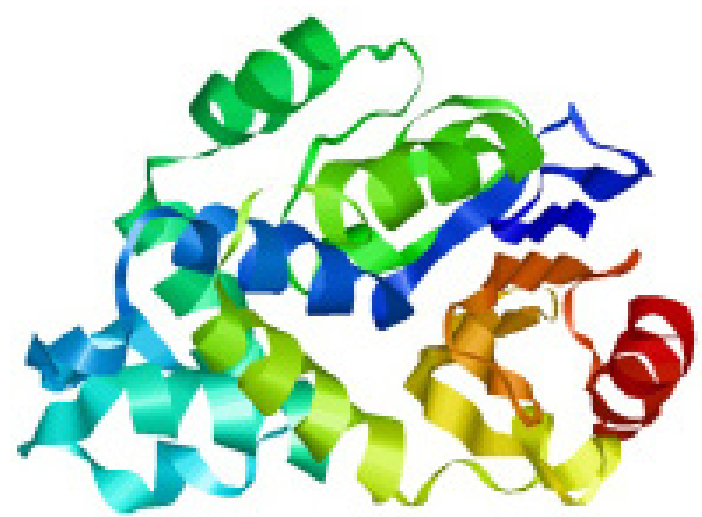

Figure 2: Modeled Beta-lactamase protein from S.sciuri shown in cartoons model.

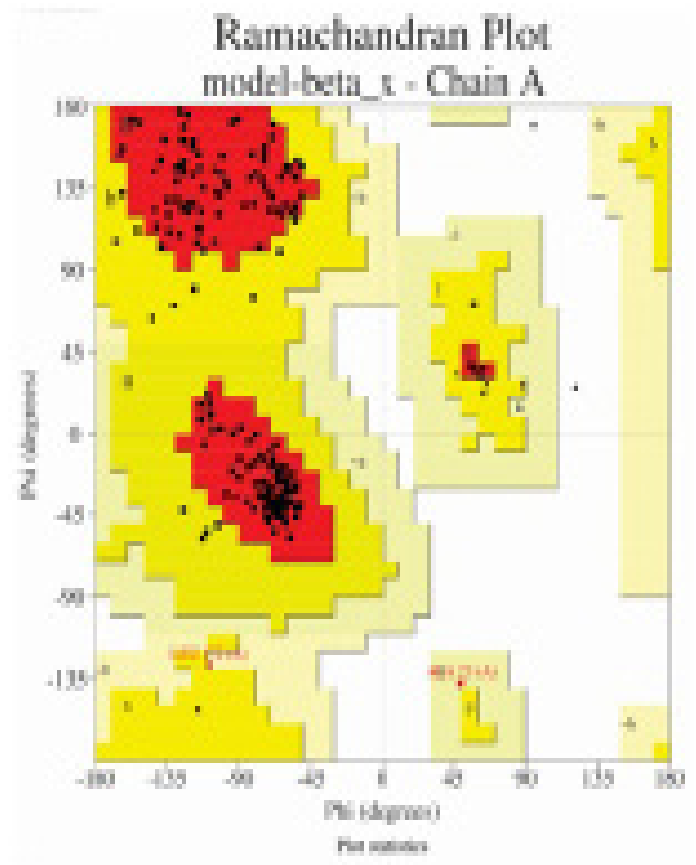

Figure 3: Ramachandran plot for the modeled Beta-lactamase protein from S.sciuri.

antibiotic Gentamycin exhibited the dock score of $-20.0425 \mathrm{~kJ} / \mathrm{mol}$. The binding of remaining compounds exhibited the docking score ranging from $-22.2263 \mathrm{~kJ} / \mathrm{mol}$ to $-0.4577 \mathrm{~kJ} / \mathrm{mol}$. The docking interactions of the best three compounds were shown in Figure 4. The weak binding interaction was observed for the compound-6. This interaction is favored by Hbond with Asn 40 and non-bonded interactions by the means of Gln41, Trp65, Asn40, Phe126, Thr109 and Phe111 with the docking score of $-0.4577 \mathrm{~kJ} / \mathrm{mol}$.

Theoretically, all the compounds showed encouraging binding and docking energy (Table 3). Among them, the 1-butyl-4-methoxybenzene and Decyl trifluoroacetate exhibited the minimum binding revealed in terms of highest dock scores of $-22.1230 \mathrm{~kJ} / \mathrm{mol}$ and $-21.2263 \mathrm{~kJ} / \mathrm{mol}$ with modeled Beta-lactamase protein from S. sciurii, when compared to that of standard antibiotic Gentamycin (dock score $-20.0425 \mathrm{~kJ} / \mathrm{mol}$ ). The interactions of the 1-butyl-4-methoxybenzene is favored by the formation of Hbond with Gln41and hydrophobic interactions with Asp39, Phe126, Asn40, Trp65, Phe111, Thr109 and Lys67. The interactions of the Decyl trifluoroacetate are favored by hbonds with Asn40 and Gln41
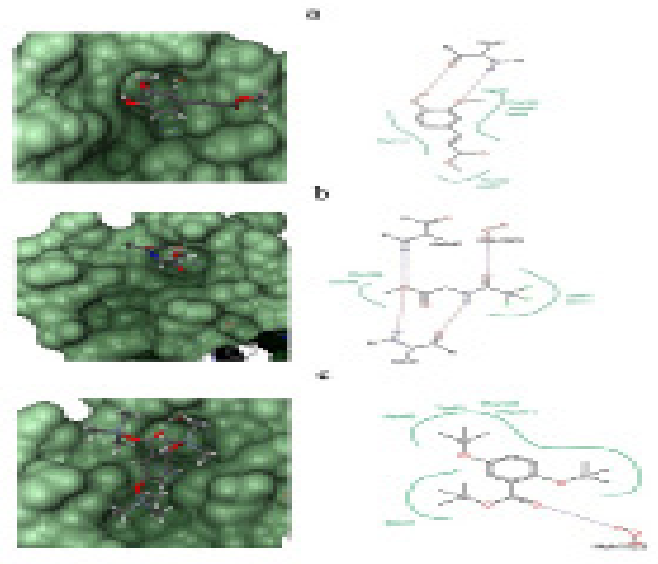

a
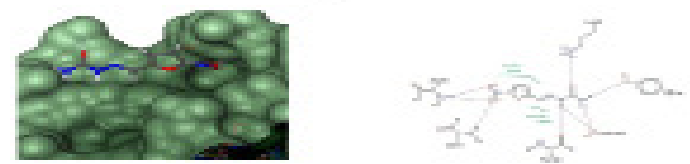

Figure 4: Docking complex and interactions of best three compounds with modeled Beta-lactamase protein from S. sciurii. (a) Docking complex and interactions of 1-butyl-4-methoxybenzene within the binding site of modeled Beta-lactamase protein from Staphylococcus sciurii, (b) Docking complex and interactions of Trans-octadec-9-ene within the binding site of modeled Beta-lactamase protein from Staphylococcus sciurii, (c) Docking complex and interactions of 2-methyloctadec-7-yne within the binding site of modeled Beta-lactamase protein from Staphylococcus sciurii and (d) Docking complex and interactions of standard antibiotic Gentamycin within the binding site of modeled Beta-lactamase protein from Staphylococcus sciurii.

Table 1: Validation of the model Beta-lactamase protein from S.sciurii by SAVES Server.

\begin{tabular}{cccccccc} 
& \multicolumn{5}{c}{ PROCHECK } & & \\
\cline { 2 - 5 } Protein & G-Factor & \multicolumn{3}{c}{ Ramachandran Plot } & \multirow{2}{*}{ ERRAT } & VERIFY- \\
\cline { 2 - 5 } & & MD & AAR & GAR & DAR & & \\
\hline $\begin{array}{c}\text { Beta- } \\
\text { lactamase }\end{array}$ & -0.13 & $92.1 \%$ & $7.0 \%$ & $0.9 \%$ & $0.0 \%$ & 83.328 & $97 \%$ \\
\hline
\end{tabular}

MFR-Most Favoured Region; AAR- Additionally allowed Regions; GAR-Generously allowed Regions; DAR-Disallowed Regions.

and non bonded interactions with Lys42, Gln41, Phe126 and Asn40. The standard antibiotic gentamycin with effective antimicrobial activity exhibited the dock score of $-20.0425 \mathrm{~kJ} / \mathrm{mol}$. This interaction is favored by Hbond formation with Lys42, Tyr130, Asn40, Gln41, Phe126 and Ser127 and also by hydrophobic interactions with Asn40, Gln41, Phe126 and Lys42. I is also observed that the 2-methyloctadec-7-yne exhibited the dock score of $-19.6326 \mathrm{~kJ} / \mathrm{mol}$, which is very close to that of the standard antibiotic Gentamycin. This interaction is favored by Hbond formation with HOH1023 and non bonded interactions with Gln41, Asn40, Trp65, Phe126 and Phe111.

\section{DISCUSSION}

GC-MS analysis of two extracts showed potent antimicrobial activity, which was described earlier ${ }^{27}$ was considered for docking. The results indicate that compound-4-Butylanisole and trans-9-Octadecene exhibited promising inhibitory activity in comparison to that of the standard antibiotic Gentamycin and 2-methyloctadec-7-yne (2-methyloctadec7 -yne) with the close margin dock score to standard drug. From the in- 
Table 2: Pockets and descriptors calculated for modeled Beta-lactamase protein from S. Sciuri.

\begin{tabular}{|c|c|c|c|c|c|}
\hline Name & $\begin{array}{c}\text { Volume } \\
{\left[\AA^{3}\right]}\end{array}$ & $\begin{array}{c}\text { Surface } \\
{\left[\AA^{2}\right]}\end{array}$ & $\begin{array}{c}\text { Lipo } \\
\text { surface } \\
{\left[\AA^{2}\right]}\end{array}$ & $\begin{array}{c}\text { Depth } \\
\text { [A]] }\end{array}$ & $\begin{array}{l}\text { Drug } \\
\text { Score }\end{array}$ \\
\hline P0 & 485.25 & 733.51 & 544.94 & 32.49 & 0.90 \\
\hline P1 & 322.11 & 354.47 & 174.67 & 19.71 & 0.76 \\
\hline $\mathrm{P} 2$ & 291.33 & 459.95 & 303.92 & 13.62 & 0.60 \\
\hline P3 & 216.00 & 350.64 & 209.16 & 11.49 & 0.45 \\
\hline $\mathrm{P} 4$ & 168.83 & 285.99 & 230.16 & 9.38 & 0.35 \\
\hline P5 & 142.14 & 342.98 & 253.82 & 10.25 & 0.32 \\
\hline P6 & 113.73 & 253.69 & 136.94 & 7.74 & 0.24 \\
\hline
\end{tabular}

vitro studies of the methanolic extracts of Garcinia imberti, the methanol extract had significant activity in terms of zone of inhibition. Interestingly, it was observed that among the docked compounds, the 1-butyl4-methoxybenzene and Decyl trifluoroacetate that exhibited the promising minimum binding and docking energy and the close related dock score for 2-methyloctadec-7-yne in comparison to that of the standard antibiotic Gentamycin where the active constituents of methanol extract as revealed by GC-MS analysis. ${ }^{27}$ Thus these compounds can be considered as good inhibitors against $S$. sciurii. Recently, molecular docking and inhibition studies on the interactions of Bacopa monnieri's potent phytochemicals against pathogenic Staphylococcus aureus. B. extract and its compound luteolin have potent antimicrobial activity against $S$. aureus. Molecular binding interaction revealed that luteolin has more specificity towards the DNA gyrase enzyme binding site and could be a novel antimicrobial compound. ${ }^{28}$ Recently molecular docking studies were carried out to investigate the antibacterial activity of Ricinus communis phytochemicals against beta-lactamase from Enterococcus faecalis and Staphylococcus aureus through molecular docking. The docking studies revealed that ferulic acid and hyperoside exhibited promising minimum docking and binding energy that is highly related to the docking score of standard antibiotic cefotaxime. ${ }^{29}$

The docking studies also implies that the conserved amino acids Glutamine $(\mathrm{Q})$ and Asparagine $(\mathrm{N})$, Lysine (L) and Phenyl alanine (F) in the active site of beta-lactamase are crucial in binding compounds with these receptor. These docking interactions implies that the $=\mathrm{O}$ (keto group) present in the compounds and $\mathrm{NH}$ (amino group) on the amino acids favors the Hbond interactions. Hence these findings throws light for the design of novel compounds with antimicrobial activity envisages that the amino acids Glutamine (Q) and Asparagine (N), Lysine (L) and Phenyl alanine (F) should be considered during its design for implying its action as a best antimicrobial compound that targets S. sciurii.

\section{CONCLUSION}

In root canal infection the chemical irrigants such as chlorohexidine and sodiumhypochlorite have been applied to treat various multi drug resistance bacterial strains. However, these chemical irrigants caused serious side effects such as tissue necrosis, gastritis and local inflammation. The phytochemicals of Garcinia imberti especially, 4 Butylanisole and trans9-Octadecene have immense potential against various beta lactamase producing multiple drug resistance bacteria. In our study Staphylococcus sciuri was used as the model organism to study the inhibitory effect of active molecules from Garcinia imberti by molecular docking. The plant phytochemicals have inhibitory effect on beta lactamase and may have potent application as natural irrigant. This natural irrigant is safe and an alternate to chemical irrigant.
Table 3: Docking scores of five most important compounds within the predicted $\mathrm{PO}$ active site of modeled Beta-lactamase protein from Staphylococcus sciuri.

\begin{tabular}{cccc}
\hline SI.No. & Compound Name & $\begin{array}{c}\text { CAS- } \\
\text { Number }\end{array}$ & $\begin{array}{c}\text { Docking score } \\
(\mathbf{k J} / \mathrm{mol})\end{array}$ \\
\hline 1 & $\begin{array}{c}\text { 4,4-dimethyl-8-methylidene-1- } \\
\text { oxaspiro[2.5] oct-6-ene }\end{array}$ & -15.1452 \\
& 1-butyl-4-methoxybenzene & $18272-84-9$ & -22.1230 \\
2 & Decyl trifluoroacetate & $333-88-0$ & -17.5766 \\
4 & Trans-octadec-9-ene & $7206-25-9$ & -21.2263 \\
5 & 2-methyloctadec-7-yne & $35354-38-2$ & -19.6326 \\
\hline
\end{tabular}

\section{ACKNOWLEDGEMENT}

The authors extend their sincere appreciation to Dr. Jaya Balaji Mano Christaine Angelo, Ganapathy Murugan and Muthu Kumar for their help in manuscript writing.

\section{CONFLICT OF INTEREST}

The authors declare that they have no conflict of interest.

\section{ABBREVIATIONS}

BLASTP: Basic Local Alignment Search Tool; DoG: Difference of Gaussian; DOPE: Discrete Optimized Protein Energy; GC-MS: Gas Chromatography and Mass Spectrophotometry; RMSD: Root-mean-square deviation; GROMOS: GROningen Molecular Simulation; PDB: Protein data bank, PROCHECK: A program to check the stereochemical quality of protein structures.

\section{REFERNCES}

1. JrSiqueira JF, Paiva SS, Rocas IN. Reduction in the cultivable bacterial populations in infected root canals by a chlorhexidine-based antimicrobial protocol. J Endod. 2007a;33(5):541-7.

2. Sakamoto M, Rocas IN, JrSiqueira JF, Benno Y. Molecular analysis of bacteria in symptomatic and symptomatic endodontic infections. Oral Microbiol Immunol. 2006;21(2):112-22.

3. Gomes BP, Lilley JD, Drucker DB. Clinical significance of dental root canal microflora. J Dent. 1996;24(1-2):47-55.

4. Sjogren U, Figdor D, Persson S, Sundqvist G. Influence of infection at the time of root filling on the outcome of endodontic treatment of teeth with apical periodontitis. Int Endod J. 1997;30(5):297-306.

5. Claudio NC, Phatak SS. Homology modeling in drug discovery: current trends and applications. Drug Discov Today. 2009;14(13-14):676-83.

6. Hillisch A, Pineda LF, Hilgenfeld R. Utility of homology models in the drug discovery process. Drug Discov Today. 2004;9(15):659-69.

7. Lengauer T, Rarey M. Computational methods for biomolecular docking. Curr Opin Struct Biol. 1996;6(3):402-6.

8. Meng EC, Shoichet BK, Kuntz ID. Automated docking with grid-based energy evaluation. J Comput Chem. 2004;13(4):505-24

9. Klebe G, Mietzner T. A fast and efficient method to generate biologically relevant conformations. J Comput Aided Mol Design. 1994;8(5):583-606.

10. Halperin I, Ma B, Wolfson H, Nussinov R. Principles of Docking: An Overview of Search Algorithms and a Guide to Scoring Functions. PROTEINS: Struct Fun Genetic. 2002;47(4):409-43.

11. Kitchen DB, Decornez H, Furr JR, Bajorath J. Docking and Scoring in virtual screening for Drug Discovery: Methods and Applications, Nature reviews. Drug Discover. 2004;3(11):935-49.

12. Kellenberger E, Rodrigo J, Muller P, Rognan D. Comparative Evaluation of Eight Docking Tools for Docking and Virtual Screening Accuracy. PROTEINS: Struct Fun Bioinformatic. 2004;57(2):225-42.

13. Zhu Y, Cleaver L, Wang W, Podoll JD, Walls S, Jolly A, et al. Tetracyclic indolines as a novel class of $\beta$-lactam-selective resistance-modifying agent for MRSA. Eur J Med Chem. 2017;125:130-42.

14. Altschul SF, Gish W, Miller W, Myers EW, Lipman DJ. Basic local alignment search tool. J Mol Biol. 1990;215(3):403-10. 
15. Berman HM, Westbrook J, Feng Z, Gilliland G, Bhat TN, et al. The protein data bank. Nucl Acids Res. 2000;28:235-42.

16. Thompson JD, Higgins DG, Gibson TJ. Clustal W: Improving the sensitivity of progressive multiple sequence alignment through sequence weighting position- specific gap penalties and weight matrix choice. Nucleic Acids Res. 1994;22(22):4673-680

17. Sali A, Blundell TL. Comparative protein modelling by satisfaction of spatial restraints. J Mol Biol. 1993;234(3):779-815.

18. Maiti R, Domselaar GHV, Zhang H, Wishart DS. Super-Pose: A simple server for sophisticated structural superposition. Nucleic Acids Res. 2004;32(suppl 2):W590-4.

19. Walter RP, Scott PH, Hunenberger IG, Tironi AE, Mark SR, Billeter JF, et al. The GROMOS biomolecular simulation program package. J Phys Chem A 1999; 103(19):3596-607.

20. Laskowski RA, MacArthur MW, Moss DS, Thornton JM. PROCHECK a program to check the stereo chemical quality of protein structure. J Appl Cryst. 1993;26(2):283-91.

21. Luthy R, Bowie JU, Eisenberg D. Assessment of protein models with threedimensional profiles. Nature. 1992;356(6364):83-5.

22. Colovos C, Yeates TO. Verification of protein structures: Patterns of nonbonded atomic interactions. Protein Sci. 1993;2(9):1511-9.
23. Khader A, Mohideen S, Sherif MA, Altaff K. In silico Sequence Analysis, Structure Prediction and Function Annotation of Melanocortin 1 Receptor Gene (MC1R) from the Guppy Poecilia reticulate. Am J Biochem Biotechnol. 2015;11(4):200-13

24. Laurie AT, Jackson RM. Q-SiteFinder: An energy-based method for the prediction of protein-ligand binding sites. Bioinformatics. 2005;21(9):1908-16.

25. Rarey M, Kramer B, Lengauer T, Klebe G. A fast flexible docking method using an incremental construction algorithm. J Mol Biol. 1996;261(3):470-89.

26. Stierand K, Maab P, Rarey M. Molecular complexes at a glance: Automated generation of two-dimensional complex diagrams. Bioinformatics. 2006;22(14):1710-6.

27. SriRamkumar SR, Sivakumar N, Angelo, JBMC. Effect of Garcinia imberti Bourd against Candida Isolates in Rootcanal Infection. JYoung Pharm. 2017;9(2):214-7.

28. Emran TB, Rahman MA, Uddin MMN, et al. Molecular docking and inhibition studies on the interactions of Bacopa monnieri's potent phytochemicals against pathogenic Staphylococcus aureus. DARU J Pharm Sci. 2015;23(1):26.

29. Umaarasu T, Padmavathy K, Thirunavukkarasu D, Rajesh SV, Shanmugam G. Molecular docking studies of Ricinus communis phytochemicals against betalactamase from Enterococcus faecalis and Staphyloccuus aureus. Asian J Clin Res. 2018;11(7):132-7.

Article History: Submission Date : 24-07-2019 ; Revised Date : 02-10-2019 ; Acceptance Date : 10-12-2019

Cite this article: Ramkumar SRS, Sivakumar N, Vijayaraghavan P. Enzymatic Inhibition of Phytochemical from Garcinia imberti on Homology Modelled Betalactamase Protein in Staphylococcus sciuri. J Young Pharm. 2020;12(1):37-41. 